
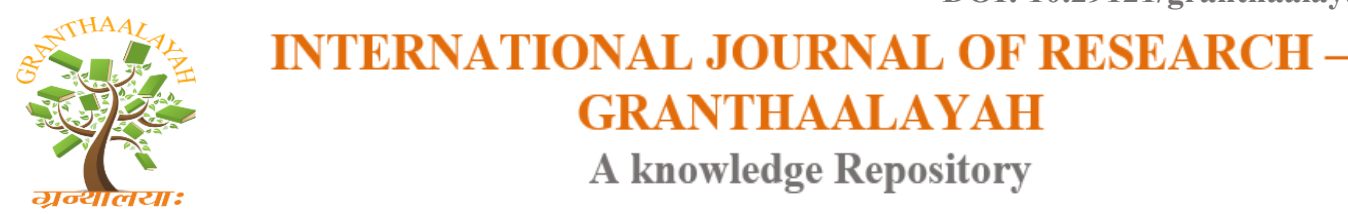

Science

\title{
FUZZY NEUTROSOPHIC RELATIONS
}

\author{
I. Arockiarani ${ }^{1}$, J. Martina Jency ${ }^{2}$ \\ ${ }^{1}$ Department of Mathematics, Nirmala College for women, Coimbatore, Tamilnadu, INDIA \\ ${ }^{2}$ Department of Mathematics, Coimbatore Institute of Engineering and Technology, Coimbatore, \\ Tamilnadu, INDIA
}

\begin{abstract}
The focus of this paper is to present the concept of fuzzy neutrosophic relations. Further we study the composition of fuzzy neutrosophic relations with the choice of t-norms and tconorms and characterize their properties.
\end{abstract}

Keywords:

Fuzzy neutrosophic set, fuzzy neutrosophic relation.

Cite This Article: I. Arockiarani, J. Martina Jency, "FUZZY NEUTROSOPHIC RELATIONS" International Journal of Research - Granthaalayah, Vol. 4, No. 2 (2016): 17-30.

\section{INTRODUCTION}

A relation is a mathematical description of a situation where certain elements of sets are related to one another in some way. It is a tool for describing correspondences between objects. The use of fuzzy relations originated from the observation that real life objects can be related to each other to certain degree. Fuzzy relations are able to model vagueness, but they cannot model uncertainty .Intuitionistic fuzzy sets, as defined by Atanassov [4], give us a way to incorporate uncertainty in an additional degree. In 1995, F.Smarandache $[13,14]$ combined the non-standard analysis with a tri component logic/set, probability theory and with philosophy and proposed the term neutrosophic which means knowledge of neutral thoughts. This neutral represents the main distinction between fuzzy and intuitionistic fuzzy logic set. Motivated by the concept which deals with non-standard analysis I.Arockiarani et al. $[1,2]$ defined the fuzzy neutrosophic set involving the concept of standard analysis. In this paper we study the properties of fuzzy neutrosophic relations in a set and the properties of the composition with different $t$-norms and $t$ conorms.

\section{PRELIMINARIES}

In order to define the fuzzy neutrosophic relations, we will use the well -known triangular norms and conorms in $[0,1]$, taking into account that as non-classical connectives. They do not satisfy 
the boolean standard identities. We will call t-norm in $[0,1]$ to every mapping $\mathrm{T}$ : $[0,1] \times[0,1] \rightarrow[0,1]$ satisfying the following properties

1. Boundary conditions, $T(x, 1)=x$ and $T(x, 0)=0$, for all $x \in[0,1]$

2. Monotony, $\mathrm{T}(\mathrm{x}, \mathrm{y}) \leq \mathrm{T}(\mathrm{z}, \mathrm{t})$ if $\mathrm{x} \leq \mathrm{z}$ and $\mathrm{y} \leq \mathrm{t}$

3. Commutative, $\mathrm{T}(\mathrm{x}, \mathrm{y})=\mathrm{T}(\mathrm{y}, \mathrm{x})$, for all $\mathrm{x}, \mathrm{y} \in[0,1]$

4. Associative, $T(T(x, y), z)=T(x, T(y, z))$, for all $x, y, z \in[0,1]$

Given a t-norm $\mathrm{T}$, we can consider the mapping $\mathrm{S}:[0,1] \times[0,1] \rightarrow[0,1] \mathrm{S}(\mathrm{x}, \mathrm{y}) \equiv 1-\mathrm{T}(1-\mathrm{x}, 1-\mathrm{y})$. This mapping $\mathrm{S}$, will be called dual $\mathrm{t}$-conorm of $\mathrm{T}$. The most important properties of $\mathrm{t}$-norms and t-conorms can be found in $[9,11]$.Here we present the following theorem with regard to the distributive property of $t$-norms and t-conorms. In this paper unless it is said in the opposite way, we will designate the t-norms and t-conorms with the greek letters $\alpha, \beta, \lambda, \rho$. Let I be a finite family of indices and $\left\{a_{i}\right\}_{i \in I},\left\{b_{i}\right\}_{i \in I}$ be number collection of $[0,1]$. For every $\alpha$ t-norm or tconorm and for every $\lambda$ t-norm or t-conorm

1. ${ }_{i}^{\alpha}\left(a_{i} \vee b_{i}\right) \geq{ }_{i}^{\alpha}\left(a_{i}\right) \vee{ }_{i}^{\alpha}\left(b_{i}\right)$

2. ${ }_{i}^{\lambda}\left(a_{i} \wedge b_{i}\right) \leq{ }_{i}^{\lambda}\left(a_{i}\right) \wedge{ }_{i}^{\lambda}\left(b_{i}\right)$ are verified.

With the result given by L.W.Fung and S.K.Ku [10] relative to the fact that $\alpha$ is an idempotent tconorm (idempotent t-norm) if and only if $\alpha=\mathrm{V}(\alpha=\Lambda)$, we get the following theorem:

\section{Theorem 2.0:}

Let $\left\{a_{i}\right\}_{i \in I},\left\{b_{i}\right\}_{i \in I}$ be two finite number families of $[0,1]$ and $\alpha, \lambda$ t-norms or $\mathrm{t}$-conorms not null. Then

${ }_{i}^{\alpha}\left(a_{i} \vee b_{i}\right)={ }_{i}^{\alpha}\left(a_{i}\right) \vee{ }_{i}^{\alpha}\left(b_{i}\right)$ if and only if $\alpha=\mathrm{V}$ ${ }_{i}^{\lambda}\left(a_{i} \wedge b_{i}\right)={ }_{i}^{\lambda}\left(a_{i}\right) \wedge{ }_{i}^{\lambda}\left(b_{i}\right)$ if and only if $\lambda=\wedge$.

\section{Definition 2.1: [2]}

A Fuzzy neutrosophic set $\mathrm{A}$ on the universe of discourse $\mathrm{X}$ is defined as $\mathrm{A}=\left\langle x, T_{A}(x), I_{A}(x), F_{A}(x)\right\rangle, x \in X$ where $T, I, F: X \rightarrow[0,1]$ and $0 \leq T_{A}(x)+I_{A}(x)+F_{A}(x) \leq 3$

\section{FUZZY NEUTROSOPHIC RELATIONS}

\section{Definition 3.1:}

A fuzzy neutrosophic set relation is defined as a fuzzy neutrosophic subset of $X \times Y$ having the form $R=\left\{\left\langle(x, y), T_{R}(x, y), I_{R}(x, y), F_{R}(x, y)\right\rangle: x \in X, y \in Y\right\}$ where $T_{R}, I_{R}, F_{R}: X \times Y \rightarrow[0,1]$ Satisfy the condition $0 \leq T_{R}(x, y)+I_{R}(x, y)+F_{R}(x, y) \leq 3 \quad \forall(x, y) \in X \times Y$. We will denote with $F N R(X \times Y)$ the set of all fuzzy neutrosophic subsets in $X \times Y$. 


\section{Definition 3.2:}

Given a binary fuzzy neutrosophic relation between $X$ and $Y$, we can define $R^{-1}$ between $Y$ and $X$ by means of $T_{R^{-1}}(y, x)=T_{R}(x, y), I_{R^{-1}}(y, x)=I_{R}(x, y), F_{R^{-1}}(y, x)=F_{R}(x, y) \forall(x, y) \in$ $X \times Y$ to which we call inverse relation of $\mathrm{R}$.

\section{Definition 3.3:}

Let $R$ and $P$ be two fuzzy neutrosophic relations between $X$ and $Y$, for every $(x, y) \in X \times Y$ We can define,

1) $R \leq P \Leftrightarrow T_{R}(x, y) \leq T_{P}(x, y), I_{R}(x, y) \leq I_{P}(x, y), F_{R}(x, y) \geq F_{P}(x, y)$

$2) R \preccurlyeq P \Leftrightarrow T_{R}(x, y) \leq T_{P}(x, y), I_{R}(x, y) \leq I_{P}(x, y), F_{R}(x, y) \leq F_{P}(x, y)$

3) $R \vee P=\left\{\left\langle(x, y), T_{R}(x, y) \vee T_{P}(x, y), I_{R}(x, y) \vee I_{P}(x, y), F_{R}(x, y) \wedge F_{P}(x, y)\right\rangle\right.$

4) $R \wedge P=\left\{\left\langle(x, y), T_{R}(x, y) \wedge T_{P}(x, y), I_{R}(x, y) \wedge I_{P}(x, y), F_{R}(x, y) \vee F_{P}(x, y)\right\rangle\right.$

5) $R^{c}=\left\{\left\langle(x, y), F_{R}(x, y), 1-I_{R}(x, y), T_{R}(x, y)\right\rangle: x \in X, y \in Y\right\}$

\section{Theorem 3.4:}

Let $\mathrm{R}, \mathrm{P}, \mathrm{Q}$ be three elements of fuzzy neutrosophic relations $(\mathrm{X} \times \mathrm{Y})$

(i) $R \leq P \Rightarrow R^{-1} \leq P^{-1} \quad(i i)(R \vee P)^{-1}=R^{-1} \vee P^{-1}(i i i)(R \wedge P)^{-1}=R^{-1} \wedge P^{-1}$

$(i v)\left(R^{-1}\right)^{-1}=R \quad(v) R \wedge(P \vee Q)=(R \wedge P) \vee(R \wedge Q), R \vee(P \wedge Q)=(R \vee P) \wedge(R \vee Q)$

(vi) $R \vee P \geq R, R \vee P \geq P, R \wedge P \leq R, R \wedge P \leq P$

(vii) If $R \geq P$ and $R \geq Q$, then $R \geq P \vee Q$

(viii) If $R \leq P$ and $R \leq Q$, then $R \leq P \vee Q$

\section{Proof:}

(i) If $R \leq P$ then $T_{R^{-1}}(y, x)=T_{R}(x, y) \leq T_{P}(x, y)=T_{P^{-1}}(y, x)$ for every $(x, y) \in X \times Y$ $I_{R^{-1}}(y, x)=I_{R}(x, y) \leq I_{P}(x, y)=I_{P^{-1}}(y, x) \forall(x, y) \in X \times Y$ and

$$
F_{R^{-1}}(y, x)=F_{R}(x, y) \geq F_{P}(x, y)=F_{P^{-1}}(y, x) \forall(x, y) \in X \times Y \text {.Hence } \quad R^{-1} \leq P^{-1}
$$

(ii) $T_{(R \vee P)^{-1}}(y, x)=T_{R \vee P}(x, y)=T_{R}(x, y) \vee T_{P}(x, y)=T_{R^{-1}}(y, x) \vee T_{P^{-1}}(y, x)$

$=T_{R^{-1} \vee P^{-1}}(y, x)$. The proofs for $I_{(R \vee P)^{-1}}(y, x)=I_{R^{-1} \vee P^{-1}}(y, x) \&$

$F_{(R \vee P)^{-1}}(y, x)=F_{R^{-1} \vee P^{-1}}(y, x)$ are done in a similar way.Hence $(R \vee P)^{-1}=R^{-1} \vee P^{-1}$.

(iii) Proof is similar to (ii) (iv) proof follows from the definition.

(v) We will use the fact that the operators $\vee$ and $\wedge$ satisfy the distributive property when they are applied to elements of $[0,1] . T_{R \wedge(P \vee Q)}(x, y)=T_{R}(x, y) \wedge\left\{T_{P}(x, y) \vee T_{Q}(x, y)\right\}$

$=\left\{T_{R}(x, y) \wedge T_{P}(x, y)\right\} \vee\left\{\left(T_{R}(x, y) \wedge T_{Q}(x, y)\right\}=T_{R \wedge P}(x, y) \wedge T_{R \wedge Q}(x, y)\right.$

$=T_{(R \wedge P) \vee(R \wedge Q)}(x, y)$. Similarly $I_{R \wedge(P \vee Q)}(x, y)=I_{(R \wedge P) \vee(R \wedge Q)}(x, y)$ and $F_{R \wedge(P \vee Q)}(x, y)=$ $F_{(R \wedge P) \vee(R \wedge Q)}(x, y)$. Hence $R \wedge(P \vee Q)=(R \wedge P) \vee(R \wedge Q)$.

The proof is analogous to the previous one, in the case of $R \vee(P \wedge Q)=(R \vee P) \wedge(R \vee Q)$.

(vi)Proof is obvious.

(vii)If $R \geq P$ and $R \geq Q, T_{R} \geq T_{P}$ and $T_{R} \geq T_{Q}, I_{R} \geq I_{P}$ and $I_{R} \geq I_{Q}, F_{R} \leq F_{P}$ and $F_{R} \leq F_{Q}$, $\Rightarrow T_{R} \geq T_{P} \vee T_{Q}, I_{R} \geq I_{P} \vee I_{Q}, F_{R} \leq F_{P} \wedge F_{Q} \Rightarrow T_{R} \geq T_{P \vee Q}, I_{R} \geq I_{P \vee Q}, F_{R} \leq F_{P \vee Q} \Rightarrow R \geq P \vee Q$ 
Similarly we can prove (viii) .We can generalize the operations between binary fuzzy neutrosophic relations $\mathrm{R}, \mathrm{Q} \in \mathrm{FNR}(\mathrm{X} \times \mathrm{Y})$. Using the well known triangular t-norms and $\mathrm{t}$ conorms in $[0,1]$. For a triangular $\mathrm{t}-$ norms $\mathrm{T}$ and its dual $\mathrm{t}-$ conforms $\mathrm{S}$, we get

$T(R, Q)=\left\{\left\langle(x, y), T\left(T_{R}(x, y), T_{Q}(x, y), T\left(I_{R}(x, y), I_{Q}(x, y)\right), S\left(F_{R}(x, y), F_{Q}(x, y)\right)\right\rangle\right\}\right.$

$S(R, Q)=\left\{\left\langle(x, y), S\left(T_{R}(x, y), T_{Q}(x, y), S\left(I_{R}(x, y), I_{Q}(x, y)\right), T\left(F_{R}(x, y), F_{Q}(x, y)\right)\right\rangle\right\}\right.$

\section{COMPOSITION OF FUZZY NEUTROSOPHIC RELATIONS}

Basing ourselves on the composition of binary IF relations in $[0,1]$ we can give the following definitions.

\section{Definition 3.5:}

Let $\alpha, \beta, \lambda, \rho$ be t-norms or t-conorms not necessarily dual two - two, $R \in F N R(X \times Y)$ and $P \in F N R(Y \times Z)$. We will call composed relation $\underset{\lambda, \rho}{2, \beta}{ }_{\lambda}^{\circ} R \in F N(X \times Z)$ to the one defined by

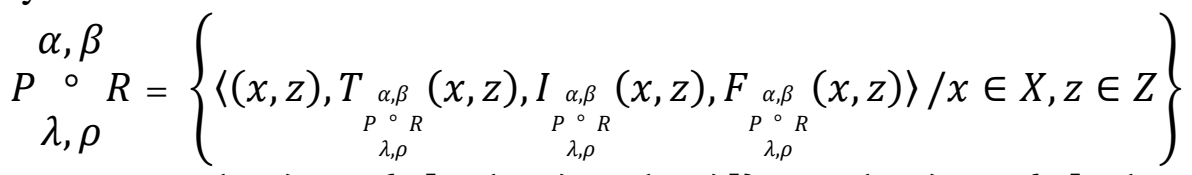

Where, $T_{\substack{\alpha, \beta \\ P 0_{R} \\ \lambda, \rho}}(x, z)={ }_{y}^{\alpha}\left\{\beta\left[T_{R}(x, y), T_{P}(y, z)\right]\right\}, I_{\substack{\alpha, \beta \\ P \circ R \\ \lambda, \rho}}(x, z)={ }_{y}^{\alpha}\left\{\beta\left[I_{R}(x, y), I_{P}(y, z)\right]\right\}$,

$$
\underset{\substack{\alpha, \beta \\ P \circ R \\ \lambda, \rho}}{F, R}(x, z)={ }_{y}^{\lambda}\left\{\rho\left[F_{R}(x, y), F_{P}(y, z)\right]\right\}
$$

Whenever $0 \leq T_{\substack{\alpha, \beta \\ P \circ R \\ \lambda, \rho}}(x, z)+I_{\substack{\alpha, \beta \\ P \circ R \\ \lambda, \rho}}(x, z)+F_{\substack{\alpha, \beta \\ P \circ R \\ \lambda, \rho}}(x, z) \leq 3 \forall(x, z) \in X \times Z$.

The choice of the t-norms and t-conorms $\alpha, \beta, \lambda, \rho$ in the previous definition, is evidently conditioned by the fulfilment of

$$
0 \leq T_{\substack{\alpha, \beta \\ P \circ R \\ \lambda, \rho}}(x, z)+\underbrace{I, R}_{\substack{\alpha, \beta \\ P \circ R \\ \lambda, \rho}}(x, z)+F_{\substack{\alpha, \beta \\ P \circ R \\ \lambda, \rho}}(x, z) \leq 3 \forall(x, z) \in X \times Z
$$

\section{Theorem 3.6:}

For each, $\in F N R(X \times Y), P \in F N R(Y \times Z)$ and $\alpha, \beta, \lambda, \rho$ any t-norms or t-conorms $\left(\begin{array}{c}\alpha, \beta \\ P \stackrel{\circ}{\alpha} R \\ \lambda, \rho\end{array}\right)^{-1}=R^{-1} \stackrel{\alpha, \beta}{\circ} P^{-1}$ is fulfilled.

Proof:

$$
\begin{aligned}
& T_{\left(\begin{array}{c}
\alpha, \beta \\
P \circ R \\
\lambda, \rho
\end{array}\right)}{ }^{-1}(z, x)=T_{\substack{\alpha, \beta \\
P \circ R \\
\lambda, \rho}}(x, z)={ }_{y}^{\alpha}\left\{\beta\left[T_{R}(x, y), T_{P}(y, z)\right]\right\}={ }_{y}^{\alpha}\left\{\beta\left[T_{R^{-1}}(y, x), T_{P^{-1}}(z, y)\right]\right\}
\end{aligned}
$$

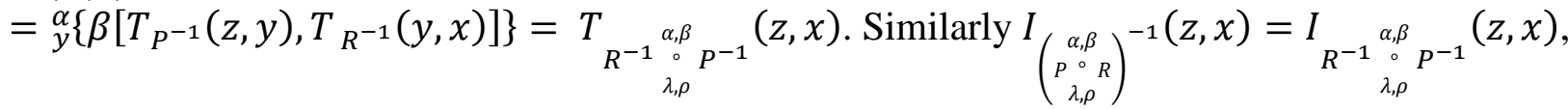




$$
\begin{aligned}
& F_{\left(\begin{array}{c}
\alpha, \beta \\
P \circ R \\
\lambda, \rho
\end{array}\right)}-1(z, x)=F_{\substack{\alpha, \beta \\
P \circ R \\
\lambda, \rho}}(x, z)={ }_{y, \rho}^{\lambda}\left\{\rho\left[F_{R}(x, y), F_{P}(y, z)\right]\right\}={ }_{y}^{\lambda}\left\{\rho\left[F_{R^{-1}}(y, x), F_{P^{-1}}(z, y)\right]\right\}
\end{aligned}
$$

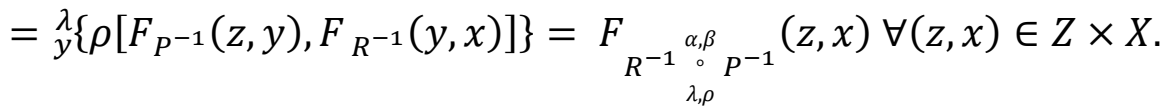

Hence $\left(\begin{array}{c}\alpha, \beta \\ P \stackrel{\circ}{\circ} R \\ \lambda, \rho\end{array}\right)^{-1}=R^{-1} \stackrel{\alpha, \beta}{\circ} P^{-1}$

\section{Theorem 3.7:}

In the conditions of definition 3.5

$$
\alpha, \beta \quad \alpha, \beta
$$

If $P_{1} \leq P_{2}$, then $P_{1} \quad \circ R \leq P_{2} \quad \circ R$, for every $\mathrm{R} \in \mathrm{FNR}$

$$
\begin{array}{cc}
\lambda, \rho & \lambda, \rho \\
\alpha, \beta & \alpha, \beta
\end{array}
$$

If $R_{1} \leq R_{2}$, then $P \quad \circ R_{1} \leq P \quad \circ R_{2}$, for every $\mathrm{P} \in \mathrm{FNR}$

$$
\begin{array}{cc}
\lambda, \rho & \lambda, \rho \\
\alpha, \beta & \alpha, \beta
\end{array}
$$

If $P_{1} \preccurlyeq P_{2}$, then $P_{1} \quad \circ R \preccurlyeq P_{2} \quad \circ R$, for every R $\in$ FNR

$$
\begin{array}{cc}
\lambda, \rho & \lambda, \rho \\
\alpha, \beta & \alpha, \beta
\end{array}
$$

If $R_{1} \preccurlyeq R_{2}$, then $P \quad \circ R_{1} \preccurlyeq P \quad \circ R_{2}$, for every $\mathrm{P} \in \mathrm{FNR}$

$$
\lambda, \rho \quad \lambda, \rho
$$

$$
\alpha, \beta \quad \alpha, \beta
$$

Let $\mathrm{R}, \mathrm{P}$ be in FNR $(\mathrm{X} \times \mathrm{X})$, if $P \leq R$, then $P \circ P \leq R{ }^{\circ} R$ are verified.

\section{Proof:}

$$
\lambda, \rho \quad \lambda, \rho
$$

$$
\begin{aligned}
& P_{1} \leq P_{2} \text { then } T_{P_{1}}(y, z) \leq T_{P_{2}}(y, z), I_{P_{1}}(y, z) \leq I_{P_{2}}(y, z) \text { and } F_{P_{1}}(y, z) \geq F_{P_{2}}(y, z) \\
& T \underset{\substack{\alpha, \beta \\
P_{1} \circ R \\
\lambda, \rho}}{\alpha, R}(x, z)={ }_{y}^{\alpha}\left\{\beta\left[T_{R}(x, y), T_{P_{1}}(y, z)\right]\right\} \leq{ }_{y}^{\alpha}\left\{\beta\left[T_{R}(x, y), T_{P_{2}}(y, z)\right]\right\}=T_{\substack{\alpha, \beta \\
P_{2} \circ o_{R} \\
\lambda, \rho}}(x, z) .
\end{aligned}
$$

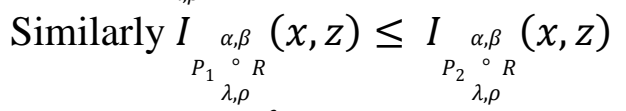

$$
\begin{aligned}
& F_{\substack{\alpha, \beta \\
P_{1} o R \\
\lambda, \rho}}(x, z)={ }_{y}^{\lambda}\left\{\rho\left[F_{R}(x, y), F_{P_{1}}(y, z)\right]\right\} \geq{ }_{y}^{\lambda}\left\{\rho\left[F_{R}(x, y), F_{P_{2}}(y, z)\right]\right\}=F_{\substack{\alpha, \beta \\
P_{2} \\
\lambda, R}}^{\alpha, \rho}(x, z) \\
& \alpha, \beta \quad \alpha, \beta \\
& \text { Therefore } \quad P_{1} \circ R \leq P_{2} \circ R \\
& \lambda, \rho \quad \lambda, \rho
\end{aligned}
$$

(ii), (iii), (iv), (v) can be proved in a similar way.

\section{Theorem 3.8:}

For any $\alpha, \beta, \lambda$ and $\rho$ t-norms or t-conorms, $R, P \in F N R(Y \times Z)$ and $Q \in F N R(X \times Y)$

$$
\alpha, \beta \quad \alpha, \beta \quad \alpha, \beta
$$

$(R \vee P) \circ Q \geq R \circ Q \vee P \circ Q$ holds

$$
\lambda, \rho \quad \lambda, \rho \quad \lambda, \rho
$$




\section{Proof:}

Starting from the points (vi), (vii), (viii) of the theorem 3.4, we get

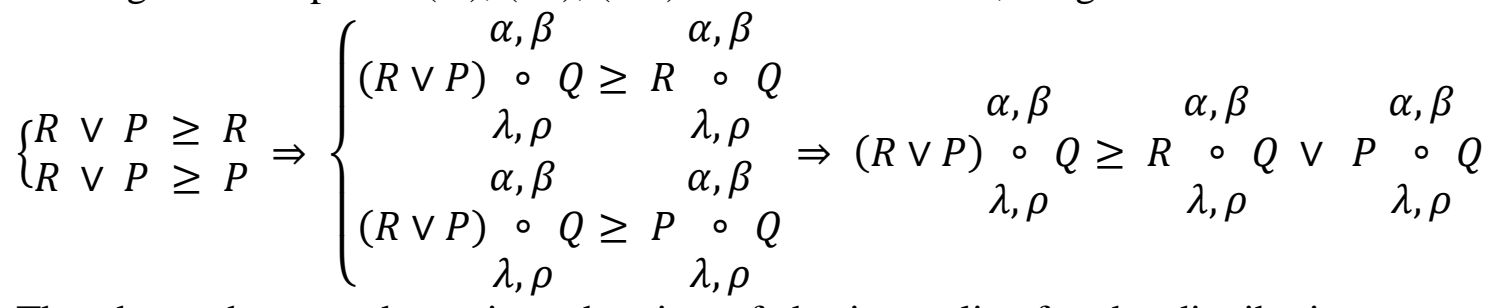

The above theorem determines the sign of the inequality for the distributive property of the composition respecting the union. Next theorem will give us a necessary and sufficient condition for the fulfilment of the equality.

\section{Theorem 3.9:}

Let R, $\mathrm{P}$ be the two elements of FNR $(Y \times \mathrm{Z}), \mathrm{Q} \in F N R(X \times Y), \alpha$ and $\lambda$ not null t-norms or

$$
\alpha, \beta \quad \alpha, \beta \quad \alpha, \beta
$$

$\mathrm{t}$-conorms. Then $(R \vee P) \circ Q=R \circ Q \vee P \circ Q$ if and only if $\alpha=\vee$ and $\lambda=\wedge$.

Proof:

$$
\lambda, \rho \quad \lambda, \rho \quad \lambda, \rho
$$

$\begin{array}{ccc}\alpha, \beta & \alpha, \beta & \alpha, \beta\end{array}$

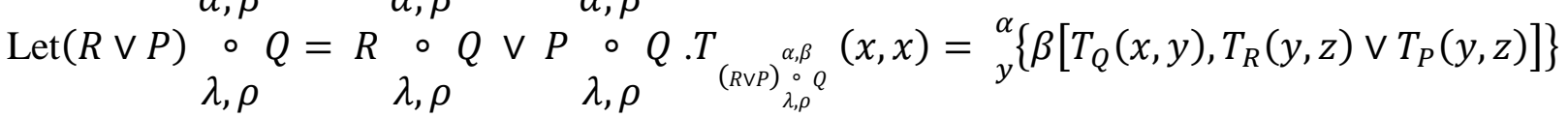

$={ }_{y}^{\alpha}\left\{\beta\left[T_{Q}(x, y), T_{R}(y, z)\right] \vee \beta\left[T_{Q}(x, y), T_{P}(y, z)\right]\right\}$. Because of the hypothesis of the theorem the result is $={ }_{y}^{\alpha}\left\{\beta\left[T_{Q}(x, y), T_{R}(y, z)\right]\right\} \vee{ }_{y}^{\alpha}\left\{\beta\left[T_{Q}(x, y), T_{P}(y, z)\right]\right\}$.

Let $\left\{a_{y}\right\}_{y \in Y}$ be any two finite family of numbers belonging to the interval $[0,1]$

If $\beta$ is t-norm, we define (for $\mathrm{x}, \mathrm{z}$ fixed and for every $\mathrm{y}$ ).

$T_{Q}(x, y)=1, T_{R}(y, z)=a_{y}, T_{P}(y, z)=b_{y}$. Then it is known that $\beta\left[T_{Q}(x, y), T_{R}(y, z)\right]=a_{y}$

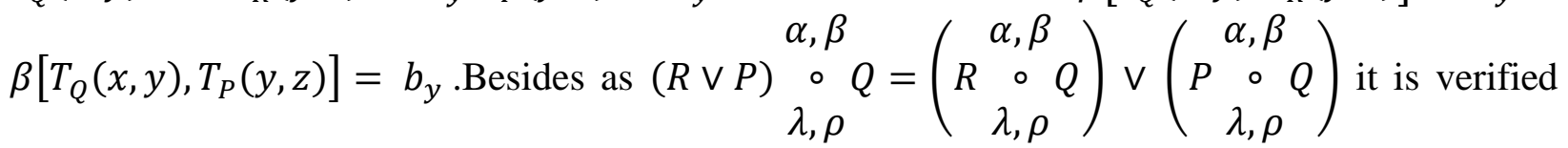

for every R, P and Q by means of hypothesis we have ${ }_{i}^{\alpha}\left(a_{i} \vee b_{i}\right)={ }_{i}^{\alpha}\left(a_{i}\right) \vee{ }_{i}^{\alpha}\left(b_{i}\right)$ and this condition verified for every $\left\{a_{y}\right\}_{y \in Y^{\prime}}\left\{b_{y}\right\}_{y \in Y}$. We have proved in the theorem 2.0, that is equivalent to $\alpha=\mathrm{V}$

(ii) If $\beta$ is $\mathrm{t}$-conorm, we define the degree of truthfulness of $\mathrm{R}, \mathrm{P}$ and $\mathrm{Q}$ as follows:

$T_{Q}(x, y)=0, T_{R}(y, z)=a_{y}, T_{P}(y, z)=b_{y}$ and with the same proceeding we conclude that verifying ${ }_{i}^{\alpha}\left(a_{i} \vee b_{i}\right)={ }_{i}^{\alpha}\left(a_{i}\right) \vee{ }_{i}^{\alpha}\left(b_{i}\right)$ as we have seen in the theorem 2.0, if and only if $\alpha=\mathrm{V}$.

In the same way we can prove the result for indeterminacy and by following the same proceeding for the falseness we can conclude that $=\Lambda$. Conversely, Let $\alpha=\mathrm{V}$ and $\lambda=\Lambda, \beta$ and $\rho$ be any $\mathrm{t}$ -

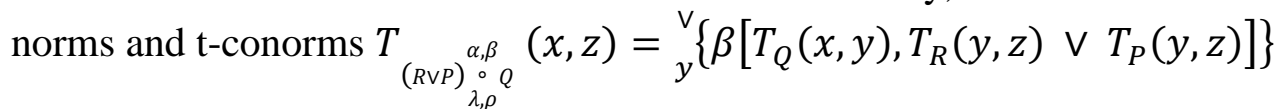
$={ }_{y}^{\vee}\left\{\beta\left[T_{Q}(x, y), T_{R}(y, z)\right] \vee \beta\left[T_{Q}(x, y), T_{P}(y, z)\right]\right\}$ 
Using the associative property of the t-conorms, we have

$={ }_{y}^{\vee}\left\{\beta\left[T_{Q}(x, y), T_{R}(y, z)\right]\right\} \vee{ }_{y}^{\vee}\left\{\beta\left[T_{Q}(x, y), T_{P}(y, z)\right]\right\}=T_{\substack{\alpha, \beta \\ R, Q \\ \lambda, \rho}}(x, z) \vee T_{\substack{\alpha, \beta \\ P \circ Q \\ \lambda, \rho}}(x, z)$

$$
\begin{aligned}
& \forall(x, z) \in X \times Z \quad \text {.Similarly } \\
& F_{\substack{\alpha, \beta \\
R \vee P)}}^{\substack{\circ, Q \\
\lambda, \rho}}(x, z)=F_{\substack{\alpha, \beta \\
R \circ Q \\
\lambda, \rho}}(x, z) \wedge F_{\substack{\alpha, \beta \\
P \circ \\
\lambda, \rho}}(x, z)
\end{aligned}
$$

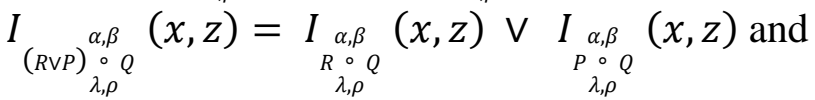

\section{Theorem 3.10:}

For every $\alpha, \beta, \lambda, \rho$ any t-norms or t-conorms and $R, P \in F N R(Y \times Z), Q \in F N R(X \times Y)$, it is

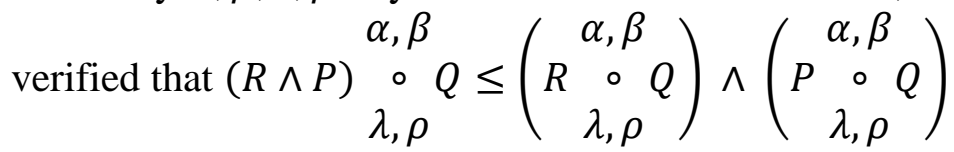

\section{Proof:}

Analogous to the one made in the theorem 3.7.

\section{Theorem 3.11:}

Let $\mathrm{R}, \mathrm{P}$ be the two elements of FNR $(Y \times \mathrm{Z}), \mathrm{Q} \in F N R(X \times Y)$ $\alpha$ different from the null t-norm and $\lambda$ different from the null t-conorm. Then $(R \wedge P)_{\lambda, \rho}^{\alpha, \beta} Q=\left(\begin{array}{c}\alpha, \beta \\ R \quad \circ \\ \lambda, \rho\end{array}\right) \wedge\left(\begin{array}{cc}\alpha, \beta \\ P & \circ \\ \lambda, \rho\end{array}\right)$ if and only if $\alpha=\wedge$ and $\lambda=\mathrm{v}$.

\section{Proof:}

The proof follows by theorem 3.9.

From the analysis of the previous theorem it is deduced that the choice of $\alpha, \beta, \lambda$ and $\rho$ t-norms or t-conorms will depend on the problem traced on each case. However the distributive equalities will demand the choice of $\vee$ and $\wedge$ for $\alpha$ and $\lambda$ or $\lambda$ and $\alpha$ respectively.

\section{Theorem 3.12:}

Let $\mathrm{Q} \in F N R(X \times Y), \mathrm{P} \in F N R(Y \times Z), \mathrm{R} \in F N R(Z \times Z) \beta$ and $\rho$ any t-norms or t-conorms.

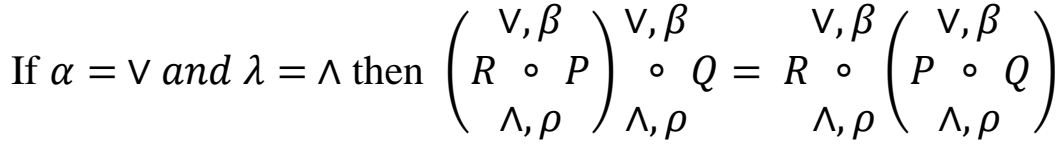

\section{Proof:}

Let $\beta$ be associative $\underset{i}{\vee} \vee_{j}^{\vee} a_{i, j}=\underset{j}{\vee} \underset{i}{\vee} a_{i, j}, \beta\left(a,{ }_{i}^{\vee_{i}} b_{i}\right)={ }_{i}^{\vee}\left(\beta\left(a, b_{i}\right)\right), \beta\left({ }_{i}^{\vee} a_{i}, b\right)={ }_{i}^{{ }^{\vee}}\left(\beta\left(a_{i}, b\right)\right)$

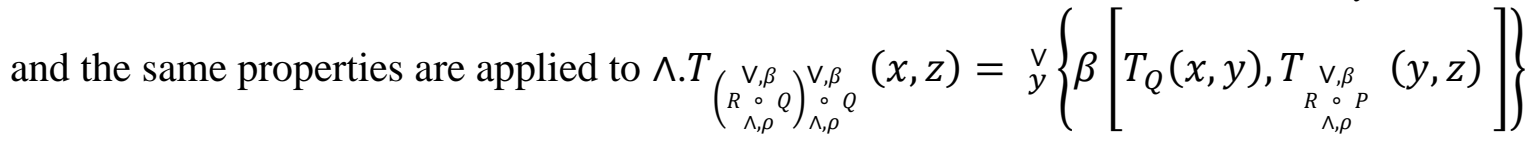




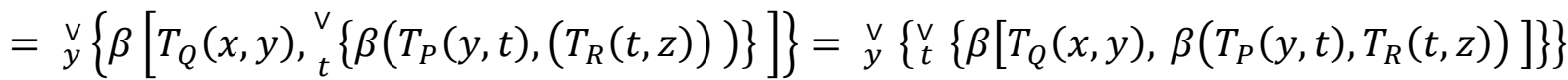

$=\quad{ }_{t}^{\vee} \quad \vee\left\{\left\{\beta\left[\beta\left[T_{Q}(x, y), T_{P}(y, t), T_{R}(t, z)\right]\right]\right\}={ }_{t}^{\vee}\left\{\beta\left[\begin{array}{c}\vee \\ y\end{array} \beta\left[T_{Q}(x, y), T_{P}(y, t), T_{R}(t, z)\right]\right]\right\}\right.$

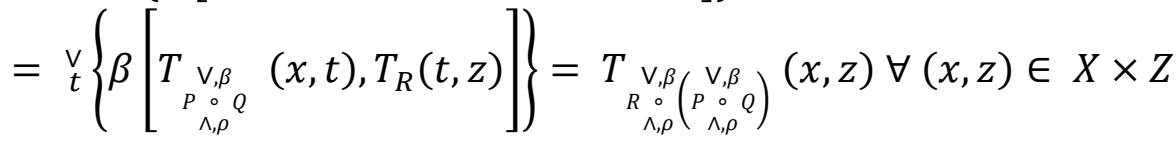

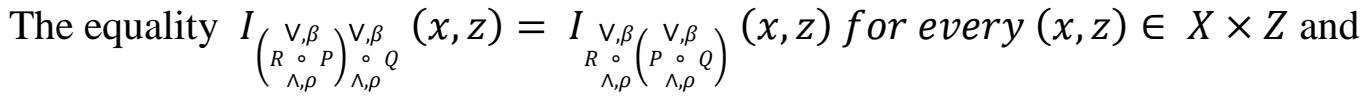

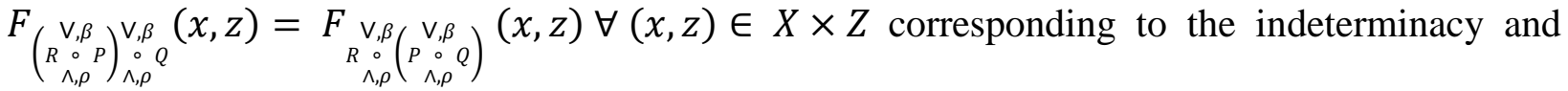
falsity are proved in a similar way.

Note:

It is convenient to state that in the notation used for composition $P_{\lambda, \rho}^{\alpha, \beta}$, the symbols $\alpha$ and $\beta$ placed up are applied to the truth value and indeterministic value and the symbols $\lambda$ and $\rho$ placed down are applied to the false value .Therefore the order of placement is very important.

\section{RELATIONS ON FUZZY NEUTROSOPHIC SETS}

\section{Definition 4.1:}

The relation $\Delta \in F N R(X \times X)$ is called the relation of identity if $\quad \forall(x, y) \in X \times X$ $T_{\Delta}(x, y)=\left\{\begin{array}{ll}1 & \text { if } x=y \\ 0 & \text { if } x \neq y\end{array}, I_{\Delta}(x, y)=\left\{\begin{array}{ll}1 & \text { if } x=y \\ 0 & \text { if } x \neq y\end{array}, F_{\Delta}(x, y)= \begin{cases}0 & \text { if } x=y \\ 1 & \text { if } x \neq y\end{cases}\right.\right.$

The complementary relation $\Delta^{c}=\nabla$ is defined by

$$
T_{\nabla}(x, y)=\left\{\begin{array}{ll}
0 & \text { if } x=y \\
1 & \text { if } x \neq y
\end{array}, I_{\nabla}(x, y)=\left\{\begin{array}{ll}
0 & \text { if } x=y \\
1 & \text { if } x \neq y
\end{array}, \quad F_{\nabla}(x, y)=\left\{\begin{array}{lll}
1 & \text { if } x=y \\
0 & \text { if } x \neq y
\end{array} .\right.\right.\right.
$$

It is evident that $\Delta=\Delta^{-1}$ and $\nabla=\nabla^{-1}$

\section{Theorem 4.2:}

Let $\alpha, \beta, \lambda, \rho$ be t-norms and t-conorms and $R \in F N R(X \times X)$

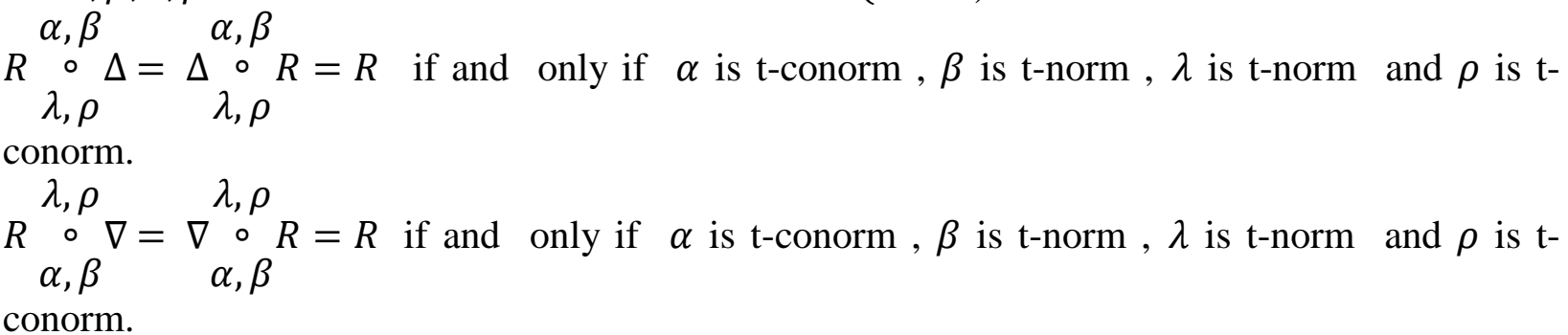




\section{Proof:}

$T_{\substack{R, \beta \\ \lambda, \rho}}^{\alpha, z}(x, z)={ }_{y}^{\alpha}\left\{\beta\left[T_{\Delta}(x, y), T_{R}(y, z)\right]\right\}=\underset{y \neq x}{\alpha}\left\{\beta\left[T_{\Delta}(x, x), T_{R}(x, z)\right], \beta\left[T_{\Delta}(x, y), T_{R}(y, z)\right]\right\}$

$=\underset{y \neq x}{\alpha}\left\{\beta\left[1, T_{R}(x, z)\right], \beta\left[0, T_{R}(y, z)\right]\right\}=\underset{y \neq x}{\alpha}\left\{\left[T_{R}(x, z), 0\right]\right\}=T_{R}(x, z),(x, z) \in X \times X$

Similarly, $I_{\alpha, \beta}(x, z)=I_{R}(x, z),(x, z) \in X \times X$

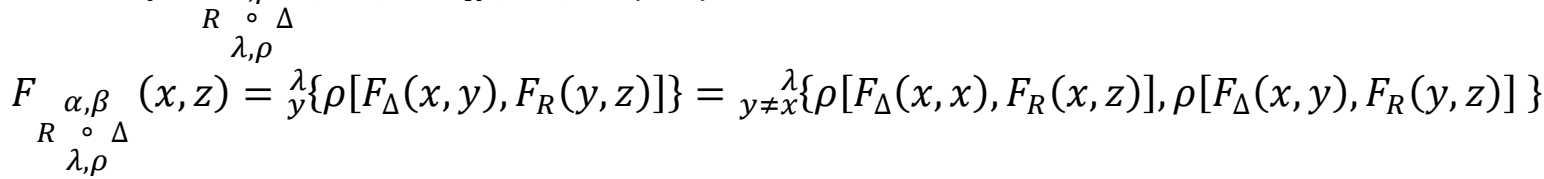

$=\underset{y \neq x}{\lambda}\left\{\rho\left[0, F_{R}(x, z)\right], \rho\left[1, F_{R}(y, z)\right]\right\}=\underset{y \neq x}{\lambda}\left\{\left[F_{R}(x, z), 1\right]\right\}=F_{R}(x, z),(x, z) \in X \times X$ $\alpha, \beta \quad \alpha, \beta$

Conversely, Suppose that $R \circ \Delta=\Delta{ }^{\circ} R=R$ it is fulfilled for each $R \in F N R(X \times X)$

Case (i):

$$
\lambda, \rho \quad \lambda, \rho
$$

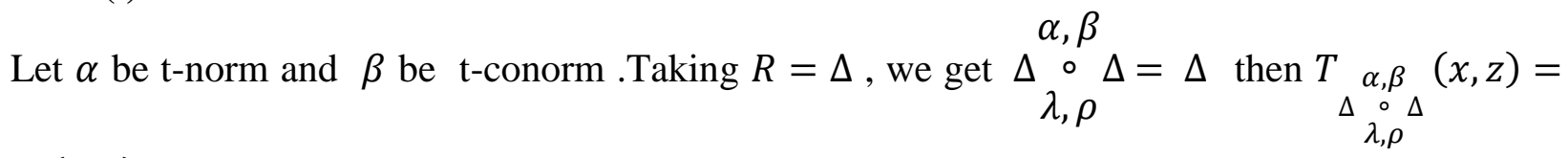

$T_{\Delta}(x, z), \forall(x, z) \in X \times X$.If $\quad x=z \quad$ then

$T_{\alpha, \beta}(x, x)={ }_{y}^{\alpha}\left\{\beta\left[T_{\Delta}(x, y), T_{\Delta}(y, x)\right]\right\}=\underset{y \neq x}{\alpha}\left\{\beta\left[T_{\Delta}(x, x), T_{\Delta}(x, x)\right], \beta\left[T_{\Delta}(x, y), T_{\Delta}(y, x)\right]\right\}$

$\Delta \stackrel{\circ}{\Delta, \rho} \Delta$

$=\underset{y \neq x}{\alpha}\{\beta[1,1], \beta[0,0]\}=\underset{y \neq x}{\alpha}\{[1,0]\}=0 \neq T_{\Delta}(x, x)=1 \forall x \in X$ Which is a contradiction.

\section{Case (ii):}

Let $\alpha$ be t-conorm and $\beta$ be t-conorm. Taking $R=\Delta$ and $x \neq z$ we have

$$
\begin{aligned}
& T_{\substack{\alpha, \beta \\
\Delta}}^{\alpha}(x, z)={ }_{y}^{\alpha}\left\{\beta\left[T_{\Delta}(x, y), T_{\Delta}(y, z)\right]\right\}=\underset{y \neq x}{\alpha}\left\{\beta\left[T_{\Delta}(x, x), T_{\Delta}(x, z)\right], \beta\left[T_{\Delta}(x, y), T_{\Delta}(y, z)\right]\right\} \\
& \Delta \stackrel{\circ}{\circ} \Delta \\
& =\underset{y \neq x}{\alpha}\left\{\beta\left[1, T_{\Delta}(x, z)\right], \beta\left[0, T_{\Delta}(y, z)\right]\right\}=\underset{\substack{\alpha \neq x \\
y \neq z}}{\alpha}\left\{\beta[1,0], \beta\left[0, T_{\Delta}(z, z)\right], \beta\left[0, T_{\Delta}(y, z)\right]\right\} \\
& =\underset{\substack{y \neq x \\
y \neq z}}{\alpha}\{\beta[1,0], \beta[0,1], \beta[0,0]\}=\underset{\substack{y \neq x \\
y \neq z}}{\alpha \neq 1}\{1,1,0\}=1 \neq T_{\Delta}(x, z)=0 \quad \forall(x, z) \in X \times X
\end{aligned}
$$

For every $(x, z) \in X \times X, T_{\substack{\Delta, \beta \\ 0 \\ \lambda, \rho}}^{\alpha, \beta}(x, z) \neq T_{\Delta}(x, z)$ if $x \neq z$

\section{Case (iii):}

Let $\alpha$ be t-norm and $\beta$ be t-conorm. Taking $R$ in the following way $T_{R}(x, y)=\left\{\begin{array}{ll}1 & \text { if } x=y \\ \neq 1 & \text { if } x \neq y\end{array}\right.$.

By means of hypothesis $T_{\substack{R, \beta \\ 0, \rho \\ \lambda, \rho}}^{\alpha}(x, x)=T_{R}(x, x)=1 \forall x \in X$ have to be fulfilled.

$T_{R, \beta}^{\alpha, \beta}(x, x)={ }_{y}^{\alpha}\left\{\beta\left[T_{\Delta}(x, y), T_{R}(y, x)\right]\right\}=\underset{y \neq x}{\alpha}\left\{\beta\left[T_{\Delta}(x, x), T_{R}(x, x)\right], \beta\left[T_{\Delta}(x, y), T_{R}(y, x)\right]\right\}$
$=\underset{y \neq x}{\alpha}\left\{\beta[1,1], \beta\left[0, T_{R}(y, x)\right]\right\}=\underset{y \neq x}{\alpha}\left\{1, T_{R}(y, x)\right\}=T_{R}(y, x) \neq 1=T_{R}(x, x)$ which is not true. 


\section{Case (iv):}

Let $\alpha$ be t-conorm and $\beta$ be t-norm .Taking $R$ in the following way $T_{R}(x, y)= \begin{cases}1 & \text { if } x=y \\ \neq 1 & \text { if } x \neq y\end{cases}$ by means of hypothesis $T_{\substack{\alpha, \beta \\ \alpha, \Delta}}^{\alpha}(x, x)=T_{R}(x, x)=1 \forall x \in X$ have to be fulfilled.

$T_{\substack{R, \beta \\ \lambda, \rho}}^{\alpha}(x, x)={ }_{y}^{\alpha}\left\{\beta\left[T_{\Delta}(x, y), T_{R}(y, x)\right]\right\}=\underset{y \neq x}{\alpha}\left\{\beta\left[T_{\Delta}(x, x), T_{R}(x, x)\right], \beta\left[T_{\Delta}(x, y), T_{R}(y, x)\right]\right\}$

$=\underset{y \neq x}{\alpha}\left\{\beta[1,1], \beta\left[0, T_{R}(y, x)\right]\right\}=\underset{y \neq x}{\alpha}\{1,0\}=1=T_{R}(x, x)$.

Hence it is proved that $\alpha$ is $\mathrm{t}$-conorm and $\beta$ is $\mathrm{t}$-norm. Proof for indeterministic functions is similar to the above.

Making a development, which is analogous to the previous one, for the falsity functions, we deduce that $\lambda$ is t-norm and $\rho$ is t-conorm.

The proof of (ii) is similar to the one made in (i) using $\nabla$.

\section{Definition 4.3:}

The relation $R \in F N R(X \times Y)$ is called

Reflexive if for every $x \in X, T_{R}(x, x)=1, I_{R}(x, x)=1, F_{R}(x, x)=0$

Anti- reflexive if for every $x \in X, T_{R}(x, x)=0, I_{R}(x, x)=0, F_{R}(x, x)=1$

(i.e,) The relation $R$ is called anti-reflexive if its complement $R^{c}$ is reflexive.

\section{Theorem 4.4:}

For every $R \in F N R(X \times Y)$, it is verified that

If $R$ is reflexive then $\Delta \leq R \quad$ (ii) If $R$ is anti-reflexive then $\Delta \geq R$

\section{Proof:}

It is the consequence of the definition 4.1 and 4.3 .

\section{Theorem 4.5:}

For $\alpha$ t-conorm, $\beta$ t-norm, $\lambda \mathrm{t}$-norm and $\rho \mathrm{t}$-conorm it is verified that

$$
\alpha, \beta
$$

If $R \in F N R(X \times Y)$ is reflexive, then $R \leq R \circ R$

$$
\lambda, \rho
$$

If $R \in F N R(X \times Y)$ is anti-reflexive, then $R \geq R \circ R$

$$
\alpha, \beta
$$




\section{Proof:}

$$
\begin{aligned}
& T_{\substack{\alpha, \beta \\
\text { o } \\
\lambda, \rho}}^{\alpha, z}(x, z)={ }_{y}^{\alpha}\left\{\beta\left[T_{R}(x, y), T_{R}(y, z)\right]\right\}=\underset{y \neq x}{\alpha}\left\{\beta\left[T_{R}(x, x), T_{R}(x, z)\right], \beta\left[T_{R}(x, y), T_{R}(y, z)\right]\right\} \\
& =\underset{y \neq x}{\alpha}\left\{\beta\left[1, T_{R}(x, z)\right], \beta\left[T_{R}(x, y), T_{R}(y, z)\right]\right\}=\underset{y \neq x}{\alpha}\left\{T_{R}(x, z), \beta\left[T_{R}(x, y), T_{R}(y, z)\right]\right\} \geq T_{R}(x, z) \\
& \text { because } \alpha \text { is t-conorm . Similarly } I_{\substack{\alpha, \beta \\
0, \rho}}(x, z) \geq I_{R}(x, z)
\end{aligned}
$$

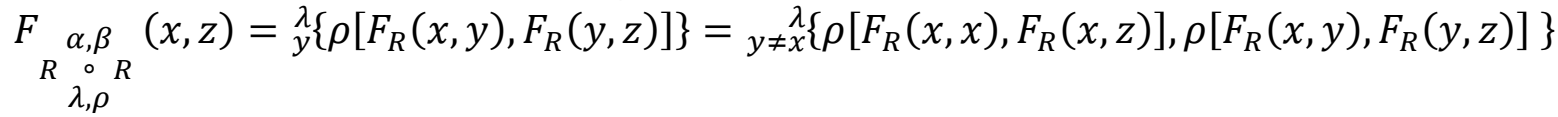

$$
\begin{aligned}
& =\underset{y \neq x}{\lambda}\left\{\rho\left[0, F_{R}(x, z)\right], \rho\left[F_{R}(x, y), F_{R}(y, z)\right]\right\}=\underset{y \neq x}{\lambda}\left\{F_{R}(x, z), \rho\left[F_{R}(x, y), F_{R}(y, z)\right]\right\} \leq F_{R}(x, z)
\end{aligned}
$$

The proof of (ii) is analogous to the proof of (i) .

\section{Example 4.6:}

This example states the existence of fuzzy neutrosophic relations which satisfy the property

$$
\alpha, \beta
$$

$R \leq R \quad \circ R$ and they are not reflexive. Let $X$ be the following set $X=\{x, y, z\}$ and $\lambda, \rho$

$R \in F N R(X \times Y)$ given by $T_{R}=\left(\begin{array}{cccc}x & x & y & z \\ x & 0.4 & 0.8 & 0.4 \\ y & 0.7 & 0.9 & 0.6 \\ z & 0.3 & 0.5 & 0.2\end{array}\right), I_{R}=\left(\begin{array}{ccccc}x & 0.3 & 0.7 & 0.2 \\ y & 0.5 & 0.8 & 0.5 \\ z & 0.1 & 0.4 & 0.1\end{array}\right)$,

$F_{R}=\left(\begin{array}{cccc}x & 0.5 & 0.3 & 0.9 \\ y & 0.4 & 0 & 0.5 \\ z & 0.8 & 0.4 & 0.6\end{array}\right)$. For $\alpha=\mathrm{V}, \beta=\wedge, \lambda=\wedge$ and $\rho=\mathrm{V}$, we have

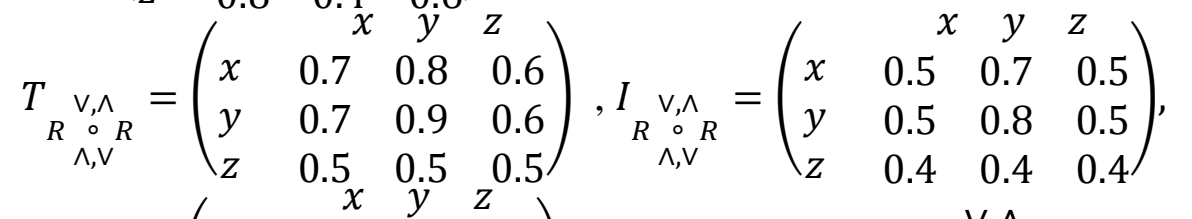

$\underset{\substack{\mathrm{V}, \wedge \\ \wedge, \mathrm{O} R}}{F}=\left(\begin{array}{cccc}x & 0.4 & 0.3 & 0.5 \\ y & 0.4 & 0 & 0.5 \\ z & 0.4 & 0.4 & 0.5\end{array}\right)$ Resulting that $R \leq R \stackrel{\vee}{\circ, \wedge} R$ not being $R$ reflexive.

\section{Theorem 4.7:}

If $R \in F N R(X \times X)$ is reflexive, $\alpha, \beta$ are t-conorms and $\lambda, \rho$ are $\mathrm{t}$-norms , then $\alpha, \beta$

$R \leq R \quad \circ R$.

$\lambda, \rho$ 


\section{Proof:}

$$
\begin{gathered}
\cos _{\substack{\alpha, \beta \\
o}}^{\lambda, \rho}(x, z)={ }_{y}^{\alpha}\left\{\beta\left[T_{R}(x, y), T_{R}(y, z)\right]\right\}=\underset{y \neq x}{\alpha}\left\{\beta\left[T_{R}(x, x), T_{R}(x, z)\right], \beta\left[T_{R}(x, y), T_{R}(y, z)\right]\right\} \\
=\underset{y \neq x}{\alpha}\left\{\beta\left[1, T_{R}(x, z)\right], \beta\left[T_{R}(x, y), T_{R}(y, z)\right]\right\}=\underset{y \neq x}{\alpha}\left\{1, \beta\left[T_{R}(x, y), T_{R}(y, z)\right]\right\}=1 \geq T_{R}(x, z)
\end{gathered}
$$

Similarly we can prove $I_{R} \underset{\substack{\alpha, \beta \\ \text { o }}}{\alpha,}(x, z) \geq I_{R}(x, z)$.

$$
\begin{aligned}
& R \underset{\lambda, \rho}{\circ}, R \\
& F_{\substack{\alpha, \beta \\
\text { o }}}^{\alpha}(x, z)=\underset{y}{\lambda}\left\{\rho\left[F_{R}(x, y), F_{R}(y, z)\right]\right\}=\underset{y \neq x}{\lambda}\left\{\rho\left[F_{R}(x, x), F_{R}(x, z)\right], \rho\left[F_{R}(x, y), F_{R}(y, z)\right]\right\} \\
& \lambda, \rho \\
& =\underset{y \neq x}{\lambda}\left\{\rho\left[0, F_{R}(x, z)\right], \rho\left[F_{R}(x, y), F_{R}(y, z)\right]\right\}=\underset{y \neq x}{\lambda}\left\{0, \rho\left[F_{R}(x, y), F_{R}(y, z)\right]\right\}=0 \leq F_{R}(x, z) \\
& \alpha, \beta
\end{aligned}
$$

Therefore $R \leq R \quad \circ R$.

$$
\lambda, \rho
$$

\section{Theorem 4.8:}

Given $R \in F N R(X \times Y)$ for $\alpha \mathrm{t}$-conorm and $\lambda \mathrm{t}$-norm, it is verified that (i) If $R$ is reflexive $\alpha, \beta$ $\alpha, \beta$

then $R \circ R$ is reflexive (ii) If $R$ is anti-reflexive then $R \circ R$ is anti reflexive

Proof: $\lambda, \rho$

$\lambda, \rho$

$$
\begin{aligned}
& T_{\substack{R, \beta \\
\text { o } \\
\lambda, \rho}}^{\alpha, x)}(x){ }_{y}^{\alpha}\left\{\beta\left[T_{R}(x, y), T_{R}(y, x)\right]\right\}=\underset{y \neq x}{\alpha}\left\{\beta\left[T_{R}(x, x), T_{R}(x, x)\right], \beta\left[T_{R}(x, y), T_{R}(y, x)\right]\right\} \\
& =\underset{y \neq x}{\alpha}\left\{\beta[1,1], \beta\left[T_{R}(x, y), T_{R}(y, x)\right]\right\}=\underset{y \neq x}{\alpha}\left\{1, \beta\left[T_{R}(x, y), T_{R}(y, z)\right]\right\}=1
\end{aligned}
$$

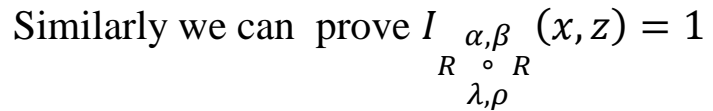

$$
\begin{aligned}
& F_{R}^{\alpha, \beta} \underset{R}{\alpha}(x, x)={ }_{y}^{\lambda}\left\{\rho\left[F_{R}(x, y), F_{R}(y, x)\right]\right\}=\underset{y \neq x}{\lambda}\left\{\rho\left[F_{R}(x, x), F_{R}(x, x)\right], \rho\left[F_{R}(x, y), F_{R}(y, x)\right]\right\}
\end{aligned}
$$

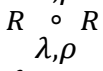

$$
\begin{aligned}
& =\underset{y \neq x}{\lambda}\left\{\rho[0,0], \rho\left[F_{R}(x, y), F_{R}(y, x)\right]\right\}=\underset{y \neq x}{\lambda}\left\{0, \rho\left[F_{R}(x, y), F_{R}(y, x)\right]\right\}=0 \text {. Therefore } \\
& \alpha, \beta \\
& R \circ R \text { is reflexive. } \\
& \lambda, \rho
\end{aligned}
$$

Proof of (ii) is similar to the one made for the reflexivity.

\section{Corollary 4.9:}

If $\quad R \in F N R(X \times X)$ is reflexive $\quad \alpha$ is t-norm and $\lambda$ is $\mathrm{t}$-conorm ,then

$$
\overbrace{\alpha, \beta \quad \alpha, \beta \quad \alpha, \beta}^{n \text { times }}
$$

$R^{(n)}=R \quad \circ R \quad \circ R \ldots \ldots . \quad \circ R$ with $\mathrm{n}=1,2,3, \ldots \ldots$ it is reflexive.

$$
\lambda, \rho \quad \lambda, \rho \quad \lambda, \rho
$$




\section{Theorem 4.10:}

Let $R_{1}$ be reflexive fuzzy neutrosophic relation in $X \times X$.Then (i) $\left(R_{1}\right)^{-1}$ is reflexive.

$R_{1} \vee R_{2}$ is reflexive for every $R_{2} \in F N R(X \times X)$ (iii) $R_{1} \wedge R_{2}$ is reflexive $\Leftrightarrow$ $R_{2} \in F N R(X \times X)$ is reflexive.

\section{Proof:}

Proof follows from the definitions.

\section{Definition 4.11:}

A reflexive closure of a relation is reflexive for every $R \in F N R(X \times X)$ is defined as $R \vee \Delta$

\section{Definition 4.12:}

A relation $R \in F N R(X \times X)$ is called symmetric if $R=R^{-1}$ that is, if for every $(x, y)$ of $X \times X, T_{R}(x, y)=T_{R}(y, x), I_{R}(x, y)=I_{R}(y, x), F_{R}(x, y)=F_{R}(y, x)$.

In a contrary manner we will say that it is asymmetric.

\section{Definition 4.13:}

Let $R$ be an element of $F N R(X \times X)$.We will say that it is antisymmetrical fuzzy neutrosophic relation if for every $(x, y)$ of $X \times X$,

$$
x \neq y \Rightarrow T_{R}(x, y) \neq T_{R}(y, x), I_{R}(x, y) \neq I_{R}(y, x), F_{R}(x, y) \neq F_{R}(y, x) .
$$

\section{Theorem 4.14:}

If $\alpha, \beta, \lambda, \rho$ are either t-norms or t-conorms and $R, P \in F N R(X \times X)$ are symmetrical then

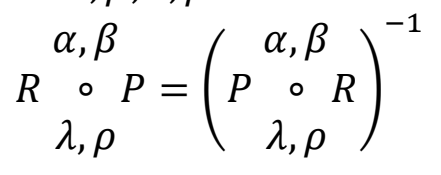

\section{Proof:}

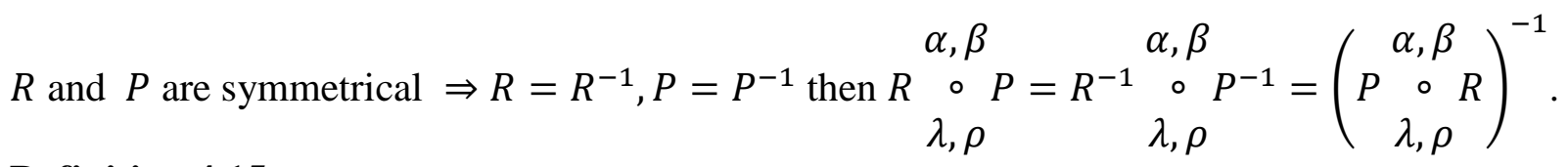

\section{Definition 4.15:}

Let us take $\alpha$ t-conorm, $\beta$ t-norm, $\lambda$ t-norm and $\rho$ t-conorm, we will say that

$$
\alpha, \beta \quad \lambda, \rho
$$

$R \in F N R(X \times X)$ is (i) transitive if $R \geq R \circ R$ (ii) c-transitive if $R \leq R \circ R$.

\section{Result 4.16:}

$$
\lambda, \rho, \beta
$$

For $\alpha \mathrm{t}$-conorm, $\beta \mathrm{t}$-norm, $\lambda \mathrm{t}$-norm and $\rho \mathrm{t}$-conorm, it is verified that 
$\alpha, \beta$

(i) If $R \in F N R(X \times X)$ is reflexive and transitive, then $R=R \circ R$

$\lambda, \rho$

$\lambda, \rho$

(ii) If $R \in F N R(X \times X)$ is anti-reflexive and c- transitive, then $R=R \circ R$

$\alpha, \beta$

\section{REFERENCES}

[1] I.Arockiarani, I.R.Sumathi and J.Martina Jency., Fuzzy Neutrosophic Soft Topological Spaces., International journal of mathematical archives ,4(10),2013., 225-238

[2] I.Arockiarani, J.Martina Jency., More on Fuzzy Neutrosophic sets and Fuzzy Neutrosophic Topological spaces,International journal of innovative research and studies .May (2014), vol 3, Issue 5,643-652.

[3] K.Atanassov, Intuitionistic fuzzy sets, Fuzzy sets and systems , 1986,20:87-96

[4] K.Atanassov,More on intuitionistic fuzzy sets , Fuzzy sets and systems, 1989,33(1):37-46.

[5] T.T .Buhaescu, Some observations on Intuitionistic fuzzy relations, Intimerat Seminar on Functional equations, 111-118.

[6] Bustince .H,P.Burillo,Structures on intuitionistic fuzzy relations, Fuzzy sets and systems, Vol.78,1996,293-303.

[7] Chakraborthy.M.K,M.Das, Studies in fuzzy relation over fuzzy subsets, Fuzzy sets and systems, Vol .9,1983,79-89.

[8] Deschrijver.G.,E.e.Kerre, On the composition of intuitionistic fuzzy relations, Fuzzy sets and systems, Vol .136,2003,333-361.

[9] D.Dubois and H.Prade, A class of Fuzzy measures based on triangular norms,Inst.J.General systems ,8,43-61(1982).

[10] L.W.Fung and K.S.Fu,An axiomatic approach to rational decision- making in a fuzzy environment ,Int:L.A.Zadeh,K.S.Fu,K.Tanaka,M.Shimura .Eds.Fuzzy sets and decision processes,Academic press, 227-256,(1975)

[11] K.Menger,Statistical metrics,Proc.N.A.S., Vol 28,1942.

[12] Mukerjee.R,Some observations on fuzzy relations over fuzzy subsets, Fuzzy sets and systems, Vol .15,1985,249-254.

[13] F.Smarandache, Neutrosophy and Neutrosophic Logic, First International Conference On Neutrosophy, Neutrosophic Logic, Set, Probability and Statistics University of New Mexico, Gallup, NM 87301,USA(2002)

[14] F.Smarandache, Neutrosophic set, a generialization of the intuituionistics fuzzy sets, Inter.J.Pure Appl.Math., 24 (2005),287 - 297. 